\title{
Emergency Ultrasound of the Chest
}

\author{
Michael Kreuter $^{\mathrm{a}}$ Gebhard Mathis $^{\mathrm{b}}$ \\ a Pneumology and Respiratory Critical Care Medicine, Thoraxklinik, University of Heidelberg, and Translational \\ Lung Research Center Heidelberg, Member of the German Center for Lung Research, Heidelberg, Germany; \\ ${ }^{b}$ Department of Internal Medicine, Landeskrankenhaus Hohenems, Hohenems, and Outpatient Clinic for Internal \\ Medicine, Rankweil, Austria
}

\section{Key Words}

Acute respiratory distress syndrome - Emergency ·

Pneumothorax · Pulmonary embolism · Thoracic ultrasound

\begin{abstract}
Thoracic ultrasound is a noninvasive and portable diagnostic tool which is highly indicated for an initial workup of thoracic emergencies. The suspicion of a pneumothorax, pneumonia, pulmonary embolism or a lung contusion after trauma can be quickly assessed using ultrasound. Main advantages are its good availability and the steep learning curve. Another advantage of thoracic sonography is that this rapid, symptom-based examination has a high sensitivity and specificity. However, a disadvantage is that only pleuraaffecting lesions or lesions visible through a sound window, e.g. an effusion or a subpleural consolidation, can be reached.
\end{abstract}

(c) 2014 S. Karger AG, Basel

\section{Introduction}

Emergency ultrasound has already exerted a significant impact on emergency medicine, e.g. for abdominal injuries after trauma, and, at present, it is the standard of care in the critical care setting, e.g. for echocardiography. However, the applicability of ultrasound to investigate thoracic emergency pathologies is still underestimated, as it is not only applicable to detect and guide drainage of pleural effusions but also to further rule out or confirm suspected pneumothorax, pulmonary embolism (PE), pneumonia, rip fractures or pericardial effusion. Still, in recent years, chest sonography has established as a useful tool in intensive care units, emergency departments and, increasingly, in the emergency room.

Main advantages are its use as a bedside modality with rapid availability due to mobile and portable devices, its capability to circumvent ionizing radiation exposure, its easy repeatability and the lack of contraindications. Thus, even children and pregnant and bedridden patients can be examined easily. Furthermore, in addition to its use as a diagnostic tool, ultrasound can also guide therapeutic, invasive procedures, e.g. drainage of pleural or pericardial effusions. Its main limitation is its dependence on the skills of the examiner, which implies that training in chest sonography is a prerequisite for proper results. Another disadvantage is that pulmonary lesions can only be detected if they are pleura based, and air artifacts in the pleural space, as long as they are not only used to detect pneu-

Previous articles in this series: 1. Herth FJF, Kreuter M: Ultrasound in pulmonology. Respiration 2014;87:1-2. 2. Gompelmann D, Herth FJF: Role of endobronchial and endoscopic ultrasound in pulmonary medicine. Respiration 2014;87:3-8.

\section{KARGER}

E-Mail karger@karger.com www.karger.com/res
(C) 2014 S. Karger AG, Basel

0025-7931/14/0872-0089\$39.50/0
Michael Kreuter, MD, FCCP, Pneumology and Respiratory Critical Care Medicine Thoraxklinik, University of Heidelberg, and Translational Lung Research Center Heidelberg, Member of the German Center for Lung Research

Amalienstrasse 5, DE-69126 Heidelberg (Germany)

E-Mail kreuter@uni-heidelberg.de 


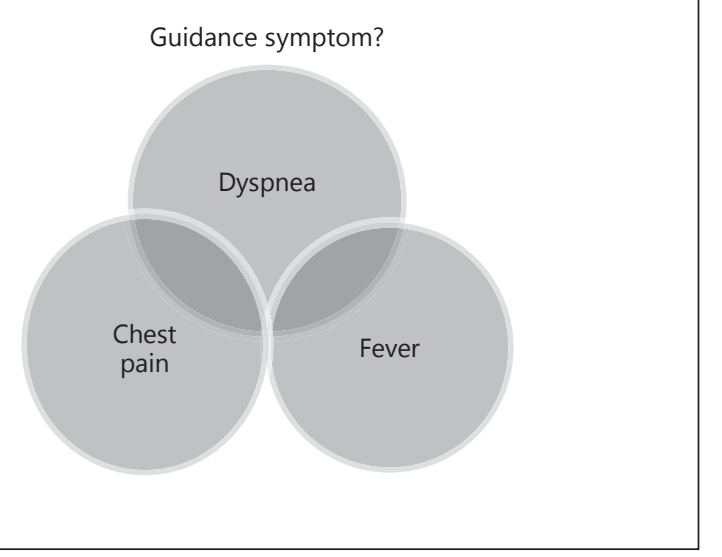

Fig. 1. The procedure of emergency ultrasound of the chest depends on the leading symptom, which might be overlapping. In point-of-care ultrasound, the first evaluation should be the site of maximum pain.

Table 1. Indications for symptom-based chest emergency ultrasound (modified according to Reissig et al. [4])

\begin{tabular}{ll}
\hline Disease entity & $\begin{array}{l}\text { Value of chest sonography } \\
\text { including echocardiography }\end{array}$ \\
\hline $\begin{array}{l}\text { Pleural and pulmonary diseases } \\
\text { Pleurisy }\end{array}$ & ++ \\
\hline Pleural effusion & ++ (combined with onsite \\
& ++ (combined with onsite \\
\hline Empyema & drainage $)$ \\
\hline Hemothorax & ++ (combined with onsite \\
\hline Pneumothorax & ++ \\
\hline PE Peripheral & + \\
\hline Pneumonia & ++ \\
\hline Pleural tumor lesions & + \\
\hline
\end{tabular}

Chest wall diseases

Rip and sternum fracture $\quad++$

Bone metastases/tumors $\quad++$

Chest wall infiltration by tumor ++

Cardiovascular disease

Acute aortic dissection

Acute coronary syndrome +

Pericardial effusion/tamponade ++

Acute heart failure ++

Peri-/myocarditis +

Heart valve disease ++

Echocardiography was included in patients with cardiovascular disease. mothorax, of a skin emphysema can limit or impair the validity of the procedure [1]. Still, chest sonography may be of help in the differential diagnoses of asthma, chronic obstructive pulmonary disease or hyperventilation, but not for the initial diagnosis of these diseases.

The primary aim of emergency ultrasound, especially in the preclinical setting, is the initial assessment of the patient to guide further (diagnostic and) therapeutic interventions. Using ultrasound in the preclinical phase after blunt abdominal trauma, Walcher et al. [2] were able to demonstrate that results of emergency ultrasound led to a change in the initially planned procedure in $22 \%$ of the cases. The purpose of imaging subsequent to the initial assessment is to aid in the diagnosis and monitoring of the disease.

\section{Symptom-Based Chest Sonography}

Most diseases of the thorax, as well as cardiac, pulmonary or other diseases, are associated with typical symptoms. Usual symptoms where thoracic ultrasound might be helpful are chest pain, dyspnea, fever and cough (fig. 1).

Life-threatening diseases associated with thoracic pain are PE, pneumothorax, esophageal rupture, acute coronary syndrome and acute dissection of the aorta. Further causes of chest pain might be pleuritis, rip fracture or tumor infiltration of the chest. In most of these cases, thoracic ultrasound including the use of echocardiography can be diagnostic (table 1) [3]. To investigate the patient, the focus of the ultrasound transducer should be the site of maximum pain.

In the diagnosis of dyspnea, ultrasound can be helpful, for example, in the detection of pleural or pericardial effusions, pneumonia or one of the previously mentioned diseases, especially PE (table 1). In addition, as a sonographic tool, echocardiography helps to identify cardiac causes such as valvular pathologies or impaired left ventricular function. Also, large intrathoracic tumors compressing central airways and/or the superior vena cava can be visualized by chest ultrasound; therefore sonography of the neck should be included in the routine sonographic evaluation of dyspneic patients.

Fever, which expresses an inflammatory response, often occurs in combination with other symptoms. Fever with chest pain can occur in chest wall abscesses, pleuritis, recurrent PE or pericarditis. It may also accompany dyspnea, e.g. in pneumonia or empyema. Solitary fever is rarely a sign of emergency for chest diseases and can be found in polyserositis, mycobacteriosis or endocarditis. 
Causes of cough, which can be diagnosed by thoracic sonography, are pneumonia, pleural diseases, pneumothorax, PE and, combined with echocardiography, cardiac pathologies [4].

\section{Sonography and Sonomorphology of Important Thoracic Emergencies}

\section{Thoracic Trauma}

Thoracic traumas account for more than half of the cases of significant road injuries, either as solitary thoracic trauma $(30 \%)$ or combined with head and thoracic injuries (18\%) [5]. Sonography of chest traumas can visualize hematomas of the chest wall, fractures of ribs and sternum, pneumothorax, hemothorax, pericardial effusions, contusion of the lung and aortic injuries. A recent trial comparing bedside chest ultrasound with clinical examination combined with chest radiography demonstrated a higher diagnostic accuracy for sonography when evaluating supine chest trauma patients, especially for pneumothorax and lung contusion [6].

The diagnosis of rib fractures can be easily made by ultrasound and is guided by the site of maximal pain [7]. Direct signs of a fracture are an interruption of the cortical reflection often coupled with a small hematoma (fig. 2). Applying slight pressure with the transducer might improve the visualization of the fracture. Indirect signs are a hematoma and a small pleural effusion. Nondislocated fractures can be identified indirectly by reverberation echoes, the so-called chimney phenomenon, which occurs at the margins of the fracture (fig. 3). Older fractures show callus formation; here, calcifications cause fine acoustic shadows. Once ossification has occurred, a continuous cortical reflex can be detected. Sonography is also helpful to identify lung contusion, where ultrasound depicts subpleural, hypoechoic, irregularly shaped lesions without air inclusions and without changes in the diameter during inspiration $[7,8]$. However, skin emphysema might limit the interpretability (fig. 4).

Paresis of the diaphragm can also be the result of a thoracic trauma. Ultrasound detects reduced mobility of the affected site and examination should therefore always be bilateral.

Examination of the thorax should accompany abdominal examination as part of the E-FAST concept (focused assessment with sonography for trauma) in order to detect rupture of the liver or spleen (fig. 5).

Emergency Ultrasound of the Chest

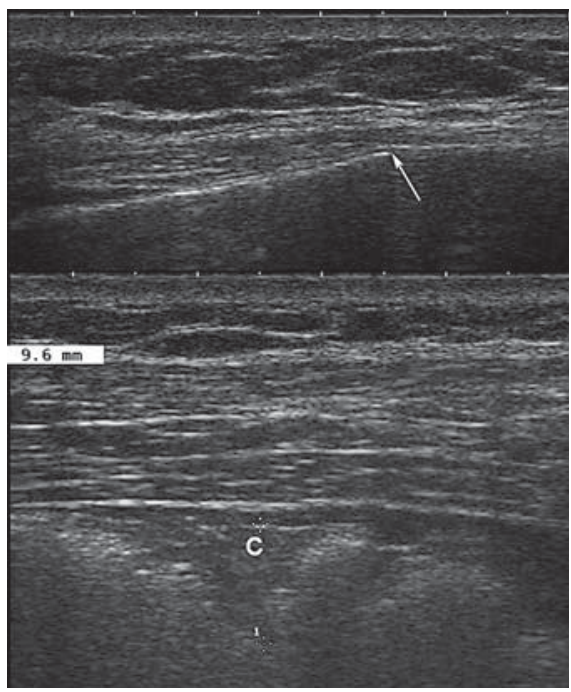

Fig. 2. Patient with a rib fracture demonstrating a minimal interruption in the cortical reflection (arrow) and signs of lung contusion $(c)$.

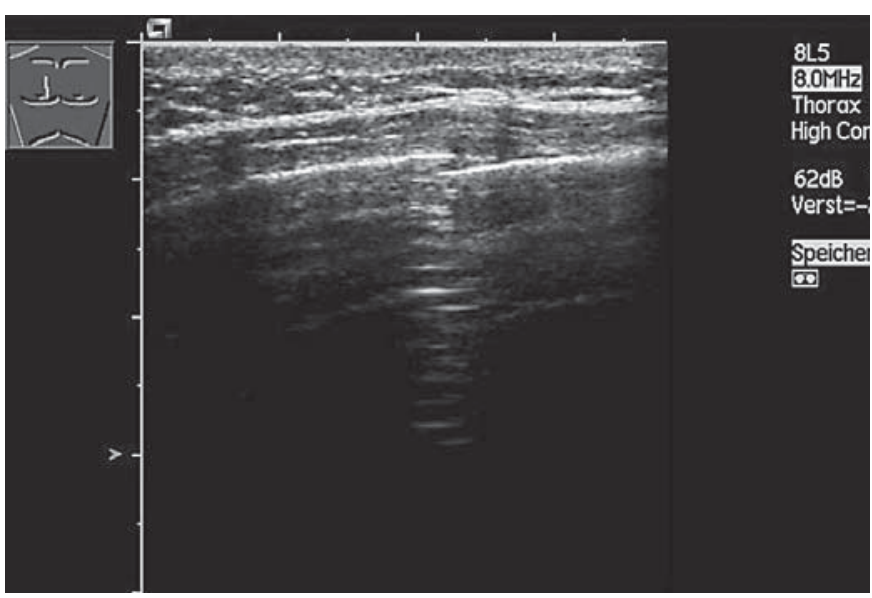

Fig. 3. Patient with a nondislocated rib fracture at the point of maximal pain showing a reverberation artifact, the so-called chimney phenomenon.

\section{Pneumothorax}

The standard procedure to diagnose a pneumothorax in most emergency departments is expiratory chest X-ray while computed tomography (CT) of the lung yields the highest sensitivity. The use of chest ultrasound to diagnose pneumothorax has long been questioned. However, in recent years, numerous clinical trials have demonstrated that pneumothorax can reliably be detected by thoracic ultrasound with a high sensitivity $(80-100 \%)$ and 


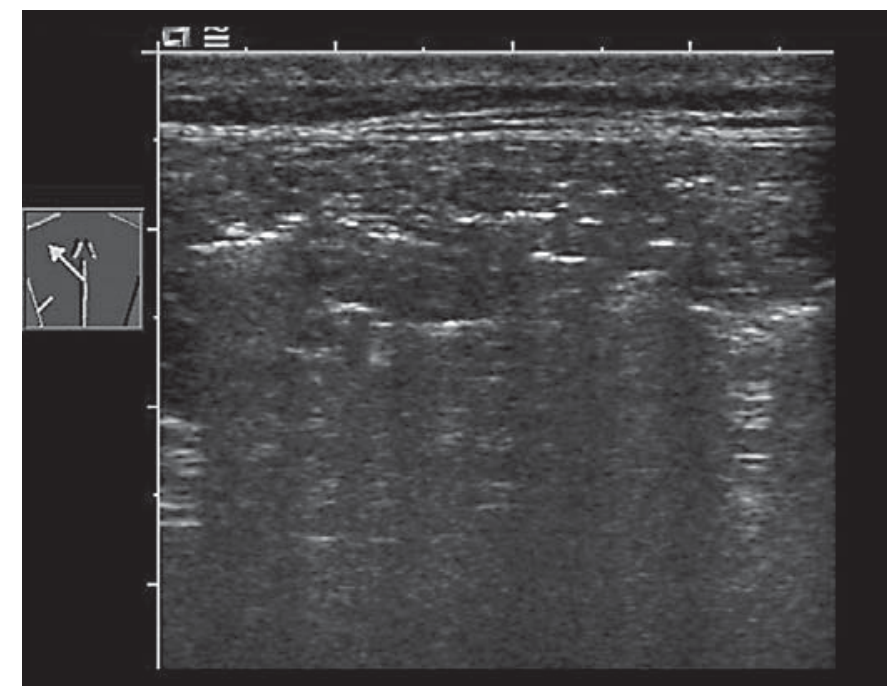

Fig. 4. Ultrasound of a patient with skin emphysema where air in the subcutis inhibits the ultrasonic investigation of distally located areas.

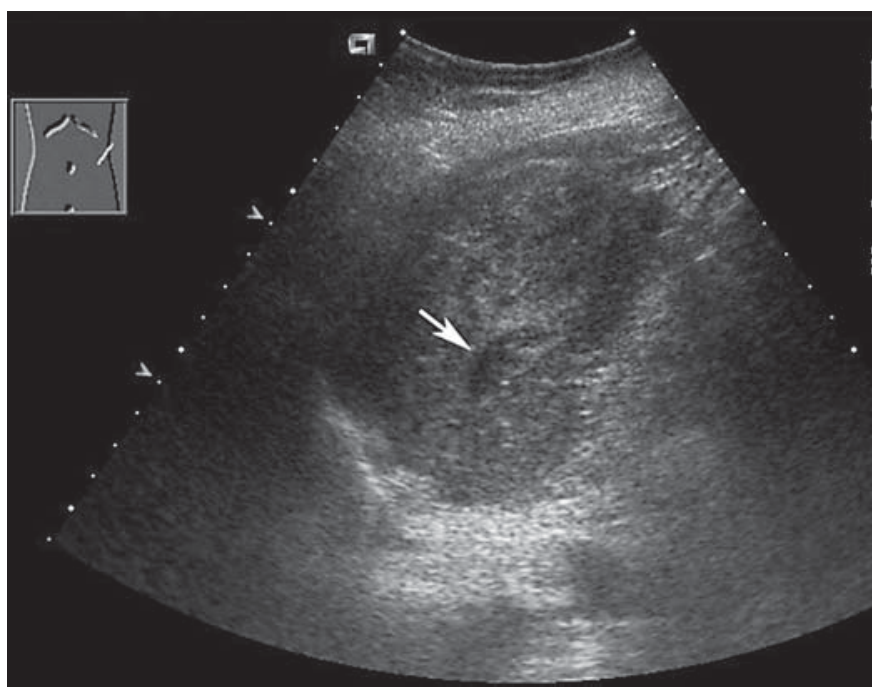

Fig. 5. Spleen rupture in a patient with rib fracture (arrow).
Fig. 6. Patient with a pneumothorax. a Healthy ventilated side with strong echogenic reflection of the lung. b Pneumothorax in the absence of sliding of the lung and augmented horizontal reverberations.
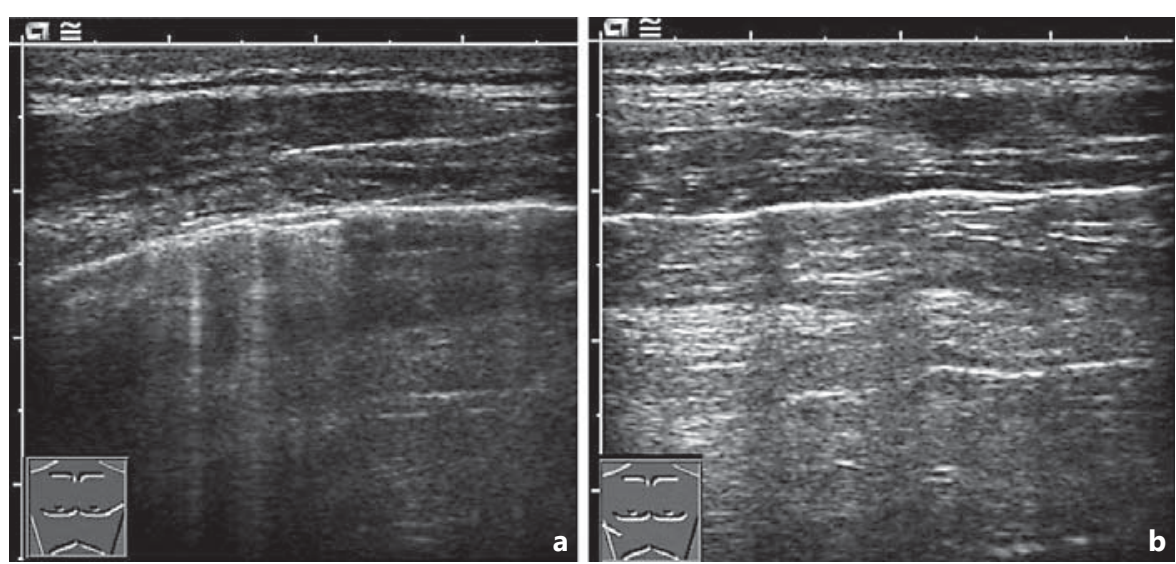

specificity (83-100\%) [3, 9-15]. Consequently, chest sonography has gained increased interest also in emergency medicine due to its high sensitivity ( $>90 \%)$ and specificity (99\%) [10, 13, 15, 16-18].

The major criterion to detect a pneumothorax sonographically is the absence of respiratory lung movement during dynamic examination, the so-called lung sliding sign. Adding power color Doppler imaging improves this examination [10]. Further diagnostic criteria are absence of B-lines, absence of lung pulse (in M-mode or power color Doppler) and finally the detection of a lung point (fig. 6,7). This reflects the border between the movement of the lung during respiration and the area of absence of the sliding sign due to the pneumothorax $[18,19]$. With the patient in the supine position, the area of interest cor- responds to the anterior part of the chest on both sides of the thorax, approximately the 3rd-4th intercostal space between the parasternal and the midclavicular lines. Of note, the results obtained have to be compared with the contralateral site. In seropneumothorax, the mobile airwater level and gas bubbles in the effusion can be visualized. One major limitation of chest sonography is that quantification of the pneumothorax is not possible. The examination is impossible in cases of subcutaneous emphysema and restricted in extreme obesity, adhesive pleural disease and emphysema of the lung $[3,10]$.

\section{Complete Opacification of Hemithorax}

The 'white out of a hemithorax' is a radiological term describing unilateral reduced radiotransparency. This is 


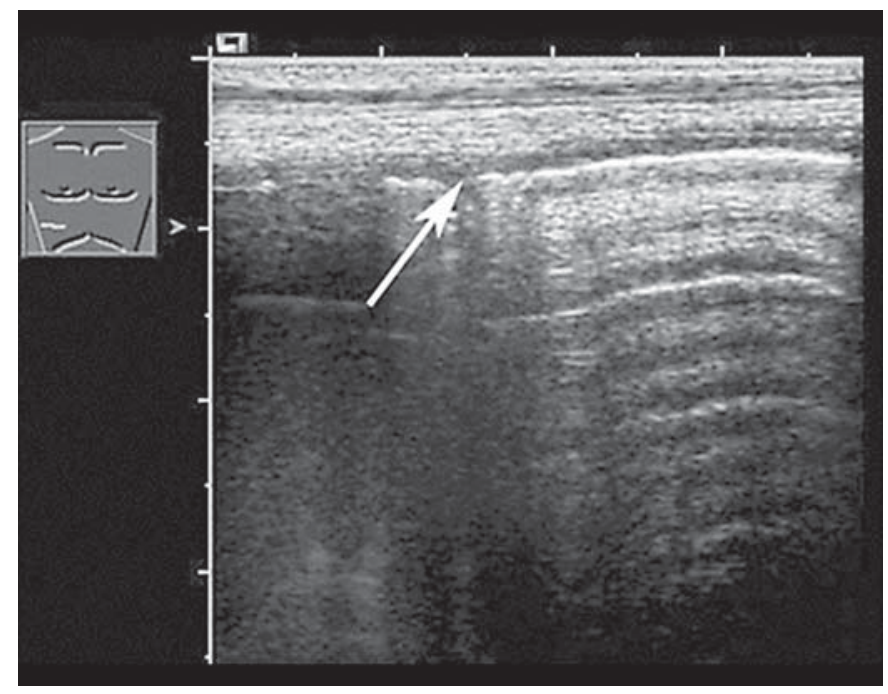

Fig. 7. Sonographic appearance of a pneumothorax by visualization of the lung point (arrow): the left part shows regular ventilated lung while on the right typical sonographic signs of a pneumothorax (reverberations) can be seen.

caused mainly by reduced air in the lungs either due to compression or due to infiltration. One can distinguish between predominantly liquid, space-occupying masses, e.g. pleural effusions, empyema, chylothorax or hemothorax, and between predominantly solid space-occupying masses, e.g. tumor-induced obstructive atelectasis. Ultrasound in predominantly liquid space-occupying masses should be stratified regarding the echogenicity of the effusion, with transudates being mainly anechoic while exudates, empyema and hemothorax have a more echogenic appearance. Septae and thickening or masses of the pleura or diaphragm, which can be observed in malignant tumors, should be noted (fig. 8). In predominantly solid masses, ultrasound should examine the homogeneity of the mass and whether an air bronchogram, e.g. in pneumonia, or a fluid bronchogram, e.g. in atelectasis or intraparenchymal pathologies such as tumors or abscesses, can be visualized. Color Doppler sonography might help to find the cause of the solid mass.

In this context, diagnostic or therapeutic puncture should be guided by sonography [20]. Especially in the diagnosis of hemothorax (hematocrit $>50 \%$ in the effusion) or empyema ( $\mathrm{pH}$ value), ultrasound is most helpful.

\section{Pericardial Effusions}

Echocardiographic examination with the sector scanner (in emergency situations, however, also possible with

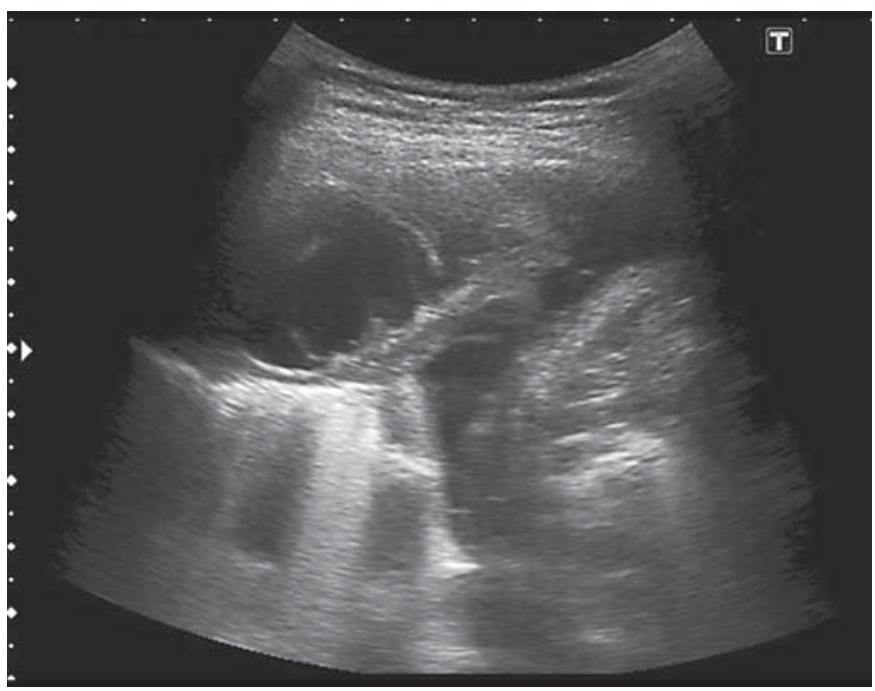

Fig. 8. Ultrasound of a pleural empyema with hypoechoic fluid and some dense internal echoes as well as septae and chambers.

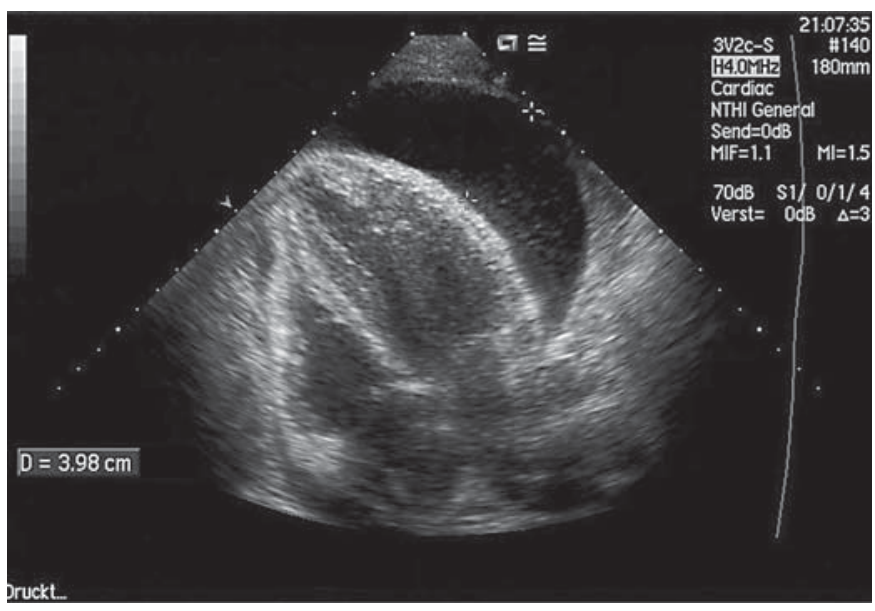

Fig. 9. Pericardial effusion.

the convex probe in the subxiphoid view during the abdominal examination as part of the E-FAST concept) allows the detection of a pericardial effusion (fig. 9). Typical signs of a pericardial effusion are a hypo- or anechoic effusion in the pericardium, and, in case of a hemodynamically relevant effusion, either right atrial or right ventricular collapse during the diastolic phase of the cardiac cycle. Further signs are an increase in the diameter of the vena cava inferior $(>2 \mathrm{~cm})$ and its variation in size throughout the respiratory cycle [21]. 


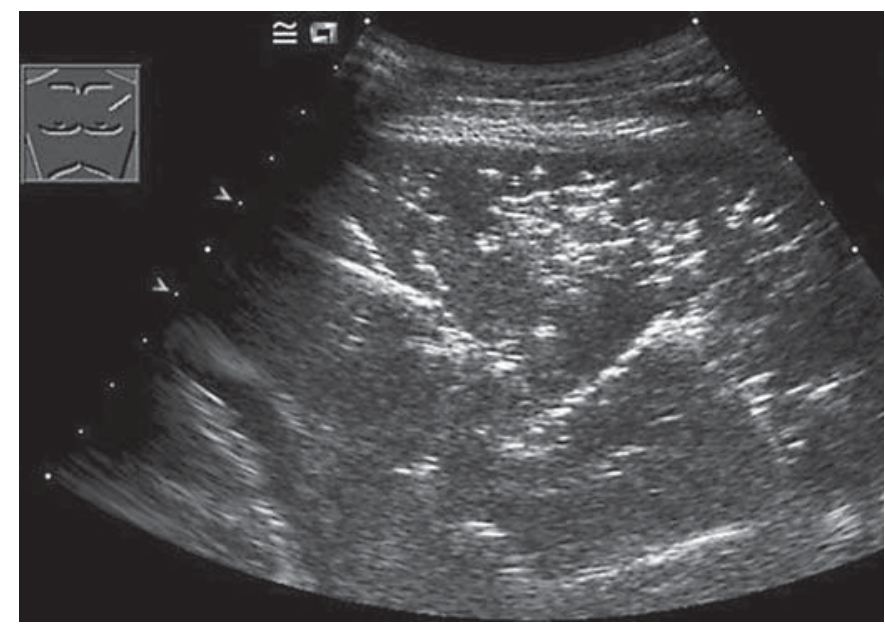

Fig. 10. Ultrasonic appearance of pneumonia with liver-like consolidation and marked bronchoaerogram.

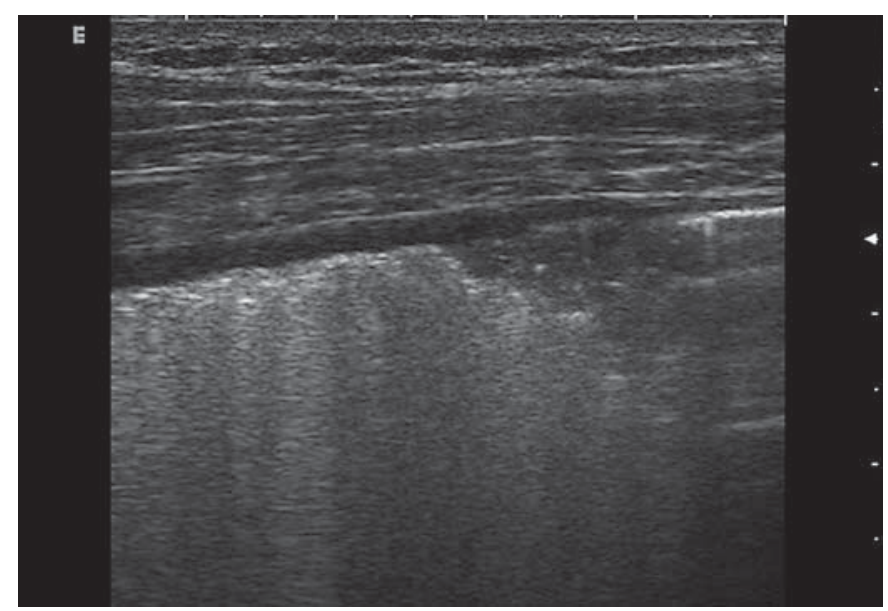

Fig. 11. Chest sonography in pleuritis: rough and interrupted reflection from the visceral pleura with small subpleural consolidation and B-lines like a focal interstitial syndrome.

\section{Pneumonia}

Community-acquired pneumonia is still one of the most common infectious diseases and an important cause of mortality and morbidity. The diagnostic evaluation of a patient with suspected pneumonia starts with the clinical evaluation followed (in distinct clinical cases) by chest radiography. However, there are several limitations of chest radiography, e.g. in critically ill patients in the emergency department who can be examined only in the supine position or preferably with bedside equipment, or regarding radiation exposure of children. Therefore, the accuracy and reliability of chest sonography have been investigated in several studies on pneumonia patients. They demonstrated a sensitivity of thoracic ultrasound $>93 \%$ with a specificity $>95 \%$. Additionally, in some of them, diagnostic accuracy for lung ultrasound and chest X-ray in detecting pneumonia corresponded [22].

Chest ultrasound depicts pneumonia as a breath-dependent, moving, hypoechoic area of varying size and shape with irregular margins together with a liver- or tissue-like echotexture (fig. 10). Also, almost similar to radiology, but with improved visualization, it shows a dynamic treelike air bronchogram with multiple small echoes within the lesion. In rare occasions, a positive fluid bronchogram is detectable by hypoechoic or echo-free tubular structures without perfusion signals. Also, pneumonia is often accompanied by pleural effusion. For further information, we would like to refer to the review by Reissig and Copetti [22] in this series.

\section{Pleuritis, Pleural Effusion and Empyema}

Pleurisy is mainly a clinical diagnosis with typical symptoms, e.g. inspiratory pain and typical auscultation. Ultrasound demonstrates a fragmented pleural reflex with small subpleural consolidations and multiple B-lines (fig. 11) [23].

Empyema, a potentially life-threatening condition, can be visualized by the detection of fluid in the pleural space with dense internal echoes, which correlates with a high cell content (fig. 8). Often, septations and chambers are present and fine needle aspiration for further analysis can be guided by ultrasound. In children, the extent of empyema can also be assessed in order to decide between conservative and surgical intervention [24]. In adults, however, CT is still the gold standard to diagnose empyema.

Even small effusions can reliably be identified using chest sonography and it is the method of choice in the diagnosis of pleural effusions. It also enables an accurate estimation of the volume of the effusion and should guide diagnostic or therapeutic drainage. For further information, we would like to refer to the article by Stigt and Groen [25] in this series on pleural effusion and ultrasound-guided chest biopsies.

\section{Pulmonary Embolism}

$\mathrm{PE}$ is a common cause of death; frequently, there is no clinical diagnosis and sometimes $\mathrm{PE}$ is oligosymptomatic. Facing the problem that CT is not always available, e.g. during night shifts and in emergencies, chest ultrasound has increasingly been used to diagnose PE, 
with reported rates for sensitivity of 70-94\% and specificity of $70-95 \%$, and an accuracy of $84-91 \%[3,14,26$, 27]. Yet, chest sonography is only diagnostic if peripheral, pleural-based lesions are detected, but these peripheral consolidations exist in $70-80 \%$ of the cases. This high value of chest ultrasound has been confirmed in a recent meta-analysis of 5 trials with more than 650 patients demonstrating a pooled sensitivity of $80 \%$ and specificity of $93 \%$. [28]. Sonographic morphology of these peripheral, pleural-based lesions, which are mainly located in the dorsal part of the lower lobe, is wedge shaped or triangular, occasionally rounded and sometimes polygonal. The lesions are small (average diameter: $1.5 \mathrm{~cm}$ ), homogeneous and hypoechoic (fig. 12). Sometimes, the central bronchial reflex is weak or absent and one might find an anechoic band of vessels on the B-mode image representing the embolus or missing/ reduced vascularization in the infarction area [29]. On contrast-assisted sonography, lung infarctions show absence of contrast enhancement. In order to be able to diagnose PE by chest sonography, 2 typical lesions should be detected [3]. Pleural effusion accompanies PE in $>50 \%$ of the cases.

About $70 \%$ of PE originate from a deep venous thrombosis of the lower extremities. Therefore, duplex sonography of leg veins with compression complements chest ultrasound with a favorable sensitivity of $90 \%$ and a specificity of $95 \%$ [30]. Direct signs of leg thrombi are visualization of the thrombus and absence of flow while indirect signs include noncompressible veins and absence of breath- dependent flow signals, for example.

Echocardiography might be also helpful as a complementary tool. In hemodynamically relevant PE, signs of right heart overload, right heart dysfunction, dilatation of the right heart and dilated pulmonary arteries can be present. Right ventricular dysfunction typically occurs in the basal heart and is more pronounced medially in case of acute right heart failure while the contractility of the apex is intact according to McConnell. An increased jet of tricuspid regurgitation and dilation of the inferior vena cava without inspiratory collapse is also often present. In central PE, sometimes the embolus can be detected directly in the pulmonary arteries. Still, a normal echocardiography cannot rule out PE due to its low sensitivity (40-70\%), which however increases in the hemodynamically instable patient [30].

Besides transthoracic and transesophageal echocardiography, a further interesting method to detect a central pulmonary embolus is endobronchial ultrasound except in emergency situations [31].

Emergency Ultrasound of the Chest

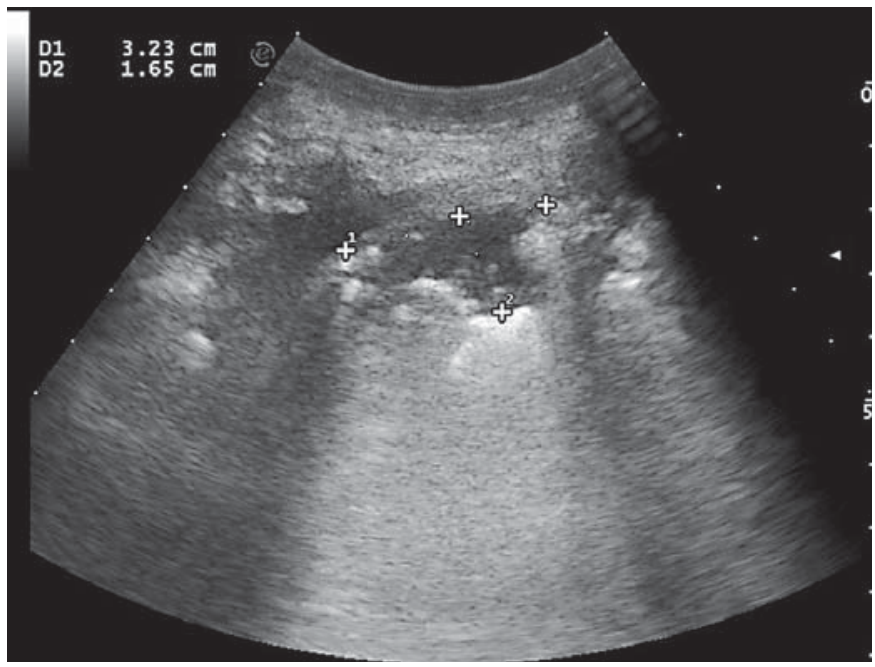

Fig. 12. Patient with pulmonary embolism: typical triangular, pleural-based echopoor consolidation.

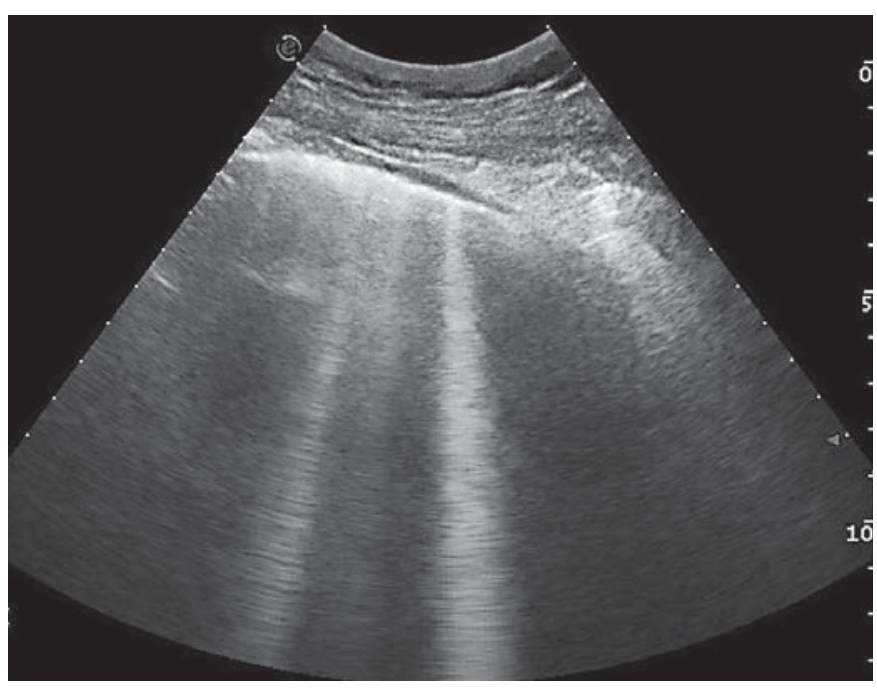

Fig. 13. Ultrasound of the chest in pulmonary embolism showing multiple, laser-like reverberations, the so called B-lines.

As a clinical algorithm for suspected PE in the cardiopulmonary-stable patient, chest ultrasound combined with compression sonography of the leg veins should be considered first. Only in case of inconspicuous thoracic ultrasound and duplex sonography of the legs, a contrast-enhanced CT is required. However, in the instable patient under suspicion of massive PE, echocardiography is the diagnostic procedure of choice and, here, normal echocardiography rules out massive PE $[3,30]$. 
Taken together, in examining patients under suspicion with PE, ultrasound is like 'killing three birds with one stone' [29]. In this context, a recent trial by Nazerianet al. [32] investigated the diagnostic validity of three-organ ultrasonography (chest, heart and leg veins) combined with the Wells score and D-dimer value. They demonstrated nicely that this combined approach was more sensitive than single-organ ultrasound and increased the accuracy of clinical pretest probability estimation in patients with suspected PE and reduced the indication for diagnostic pulmonary CT angiography.

\section{Pulmonary Edema und Acute Respiratory Distress Syndrome}

B-lines, formerly known as comet tail artifacts, are hyperechoic reverberation artifacts caused by edematous interlobular septa within the lung that arise from the visceral pleura and their moving is breath dependent (fig. 13). Initially, B-lines are found in the lung basis but extend with increasing capillary venous pressure to the superior lung and are characteristic signs of the interstitial syndrome typically present in cardiopulmonary ede$\mathrm{ma}$ and in the acute respiratory distress syndrome (ARDS). The following findings help to distinguish cardiopulmonary edema from ARDS; in cardiac edema, the above-described findings are usually bilateral and symmetric and pleural line abnormalities are only occasionally found, whereas in ARDS B-lines have a nonhomogeneous distribution and marked pleural line abnormalities [33-35]. However, B-lines and the interstitial syndrome are not pathognomonic for pulmonary edema or ARDS, but may also be found in pulmonary edema due to other reasons, interstitial pneumonia, pneumonitis and diffuse parenchymal lung diseases. To overcome some of these limitations, while chest ultrasound gives hints to pulmonary decompensation such as pulmonary edema, it can help to exclude cardiac causes of dyspnea, e.g. in obstructive lung disease B-lines are never present.

\section{Central Venous Catheterization}

In emergency situations, especially in the hemodynamically instable patient or patients in shock, the placement of a central vein catheter is generally challenging. Here, ultrasound-assisted venous catheterization enables the safe insertion of a central vein catheter as a real-time ultrasound-guided procedure, e.g. into the internal jugular or subclavian vein. Moreover, it is particularly helpful in patients in whom landmarks are not visible and not palpable [36].

\section{Conclusion}

Thoracic ultrasound adds significant value in diagnosing patients with suspected pneumothorax, $\mathrm{PE}$, pneumonia, rip fractures and pericardial effusion. Therefore, it should be implemented into emergency diagnostic procedures of patients presenting with chest symptoms and patients after chest trauma.

\section{Financial Disclosure and Conflicts of Interest}

Nothing to disclose.

\section{References}

1 Mathis G: Emergency ultrasound on the chest (in German). Praxis (Bern 1994) 2006;95: 638-643.

2 Walcher F, Weinlich M, Conrad G, et al: Prehospital ultrasound imaging improves management of abdominal trauma. Br J Surg 2006; 93:238-242.

-3 Reissig A, Copetti R, Kroegel C: Current role of emergency ultrasound of the chest. Crit Care Med 2011;39:830-845.

4 Reissig A, Pörner T, Hocke M, Kroegel C: Trans thoracic ultrasound in the diagnosis of cough, dyspnea and chest pain. Pneumologie 2007; 125:20-40.

5 Ndiaye A, Chambost M, Chiron M: The fatal injuries of car drivers. Forensic Sci Int 2009; 184:21-27.
6 Hyacinthe AC, Broux C, Francony G, Genty C, Bouzat P, Jacquot C, Albaladejo P, Ferretti GR, Bosson JL, Payen JF: Diagnostic accuracy of ultrasonography in the acute assessment of common thoracic lesions after trauma. Chest 2012;141:1177-1183.

7 Wüstner A, Gehmacher O, Hämmerle S, Schenkenbach C, Häfele H, Mathis G: Ultrasound diagnosis in blunt thoracic trauma (in German). Ultraschall Med 2005;26:285-290.

8 Rocco M, Carbone I, Morelli A, Bertoletti L, Rossi S, Vitale M, Montini L, Passariello R, Pietropaoli P: Diagnostic accuracy of bedside ultrasonography in the ICU: feasibility of detecting pulmonary effusion and lung contusion in patients on respiratory support after severe blunt thoracic trauma. Acta Anaesthesiol Scand 2008;52:776-784.
9 Garofalo G, Busso M, Perotto F, De Pascale A, Fava C: Ultrasound diagnosis of pneumothorax. Radiol Med 2006;111:516-525.

10 Kreuter M, Eberhardt R, Wenz H, Schmitteckert H, Heussel CP, Herth F: Diagnostic value of transthoracic ultrasound compared to chest radiography in the detection of a post-interventional pneumothorax (in German). Ultraschall Med 2011;2(suppl 2):E20E23.

11 Reißig A, Kroegel C: Accuracy of transthoracic sonography in excluding postinterventional pneumothorax and hydropneumothorax. Comparison to chest radiography. Eur J Radiol 2005;53:463-470. 
$\checkmark 12$ Sartori S, Tombesi P, Trevisani L, Nielsen I, Tassinari D, Abbasciano V: Accuracy of transthoracic sonography in detection of pneumothorax after sonographically guided lung biopsy: prospective comparison with chest radiography. AJR Am J Roentgenol 2007;188:37-41.

13 Soldati G, Testa A, Sher S, Pignataro G, La Sala M, Silveri NG: Occult traumatic pneumothorax - diagnostic accuracy of lung ultrasonography in the emergency department. Chest 2008;133:204-211.

14 Volpicelli G, Elbarbary M, Blaivas M, Lichtenstein DA, Mathis G, Kirkpatrick AW, Melniker L, Gargani L, Noble VE, Via G, Dean A Tsung JW, Soldati G, Copetti R, Bouhemad B, Reissig A, Agricola E, Rouby JJ, Arbelot C, Liteplo A, Sargsyan A, Silva F, Hoppmann R, Breitkreutz R, Seibel A, Neri L, Storti E, Petrovic $\mathrm{T}$; International Liaison Committee on Lung Ultrasound (ILC-LUS) for International Consensus Conference on Lung Ultrasound (ICC-LUS): International evidencebased recommendations for point-of-care lung ultrasound. Intensive Care Med 2012;38: 577-591.

-15 Zhang M, Liu ZH, Yang JX, Gan JX, Xu SW, You XD, Jiang GY: Rapid detection of pneumothorax by ultrasonography in patients with multiple trauma. Crit Care 2008 10:R112.

16 Blaivas M: Inadequate needle thoracostomy rate in the prehospital setting for presumed pneumothorax: an ultrasound study. J Ultrasound Med 2010;29:1285-1289.

17 Kirkpatrick AW, Sirois M, Laupland KB, Liu D, Rowan K, Ball CG, Hameed SM, Brown R, Simons R, Dulchavsky SA, Hamiilton DR, Nicolaou S: Hand-held thoracic sonography for detecting post-traumatic pneumothoraces: the Extended Focused Assessment with Sonography for Trauma (EFAST). J Trauma 2004;57:288-295

18 Volpicelli G: Sonographic diagnosis of pneumothorax. Intensive Care Med 2011;37:224232.
19 Lichtenstein D, Meziere G, Bidermann P, Gepner A: The 'lung point': an ultrasound sign specific to pneumothorax. Intensive Care Med 2000;26:1434-1440.

20 Stigt JA, Oostdijk AH, Boers JE, van den Berg JW, Groen HJ: Percutaneous ultrasoundguided biopsies in the evaluation of thoracic tumours after PET-CT: a prospective diagnostic study. Respiration 2012;83:45-52.

21 Goodman A, Perera P, Mailhot T, Mandavia $D$ : The role of bedside ultrasound in the diagnosis of pericardial effusion and cardiac tamponade. J Emerg Trauma Shock 2012;5:7275.

22 Reissig A, Copetti R: Lung ultrasound in community-acquired pneumonia and in interstitial lung diseases. Respiration DOI: $10.1159 / 000357449$.

23 Gehmacher O, Kopf A, Scheier M, Bitschnau R, Wertgen T, Mathis G: Ist eine Pleuritis sonographisch darstellbar? Ultraschall Med 1997; 18:214-219.

24 Ramnath RR, Heller RM, Ben-Ami T, et al: Implications of early sonographic evaluation of parapneumonic effusions in children with pneumonia. Pediatrics 1998;101:68-71.

25 Stigt JA, Groen HJ: Pleural effusion and ultrasound-guided chest biopsies. Respiration, in press. ((bitte so setzen, DOI noch unbekannt))

26 Squizzato A, Rancan E, Dentali F, Bonzini M, Guasti L, Steidl L, Mathis G, Ageno W: Diagnostic accuracy of lung ultrasound for pulmonary embolism: a systematic review and metaanalysis. J Thromb Haemost 2013;11:12691278.

27 Reissig A, Heyne JP, Kroegel C: Ancillary lung parenchymal findings at spiral CT scanning in pulmonary embolism. Eur J Radiol 2004; 49:250-257.

28 Niemann T, Egelhof T, Bongartz G: Transthoracic sonography for the detection of pulmonary embolism - a meta-analysis. Ultraschall Med 2009;30:150-156.
29 Mathis G, Blank W, Reissig A, Lechleitner P, Reuss J, Schuler A, Beckh S: Thoracic ultrasound for diagnosing pulmonary embolism: a prospective multicenter study of 352 patients. Chest 2005;128:1531-1538.

30 Torbicki A, Perrier A, Konstantinides S, Agnelli G, Galiè N, Pruszczyk P, Bengel F, Brady AJ, Ferreira D, Janssens U, Klepetko W, Mayer E, Remy-Jardin M, Bassand JP; ESC Committee for Practice Guidelines (CPG): Guidelines on the diagnosis and management of acute pulmonary embolism: the Task Force for the Diagnosis and Management of Acute Pulmonary Embolism of the European Society of Cardiology (ESC). Eur Heart J 2008;29: 2276-2315.

-31 Aumiller J, Herth FJ, Krasnik M, Eberhardt R Endobronchial ultrasound for detecting central pulmonary emboli: a pilot study. Respiration 2009;77:298-302.

32 Nazerian P, Vanni S, Volpicelli G, Gigli C, Zanobetti $\mathrm{M}$, Bartolucci $\mathrm{M}$, Ciavattone $\mathrm{A}, \mathrm{La}-$ morte A, Veltri A, Fabbri A, Grifoni S: Accuracy of point-of-care multiorgan ultrasonography for the diagnosis of pulmonary embolism. Chest, Epub ahead of print.

33 Copetti R, Soldati G, Copetti P: Chest sonography: a useful tool to differentiate acute cardiogenic pulmonary edema from acute respiratory distress syndrome. Carciovasc Ultrasound 2008;6:16-25.

34 Gargani L, Frassi F, Soldati G, Tesorio P, Gheorghiade M, Picano E: Ultrasound lung comets for the differential diagnosis of acute cardiogenic dyspnoea: a comparison with natriuretic peptides. Eur J Heart Fail 2008;10: 70-77.

35 Volpicelli G, Mussa A, Garofalo G, Cardinale L, Casoli G, Perotto F, Fava C, Frascisco M: Bedside lung ultrasound in the assessment of alveolar-interstitial syndrome. Am J Emerg Med 2006;24:689-696.

36 Hrics P, Wilber S, Blanda MP, Gallo U: Ultrasound-assisted internal jugular vein catheterization in the ED. Am J Emerg Med 1998;16: 401-403. 